\title{
Left hand, but not right hand, reaching is sensitive to visual context
}

\author{
Jos J. Adam • Rick Müskens $\cdot$ Susan Hoonhorst • \\ Jay Pratt $\cdot$ Martin H. Fischer
}

Received: 4 January 2010/Accepted: 28 February 2010/Published online: 19 March 2010

(C) The Author(s) 2010. This article is published with open access at Springerlink.com

\begin{abstract}
Recently, it has been reported that grasping with the left hand is more vulnerable to visual size illusions than grasping with the right hand. The present study investigated whether this increased sensitivity of the left hand for visual context extends to reaching. Left- and righthanded participants reached for targets embedded in two different visual contexts with either left or right hands. Visual context was manipulated by presenting targets either in a blank field or within an array of placeholders marking possible target locations. Regardless of handedness, the presence of placeholders affected left hand, but not right hand, reaching by improving end-point accuracy and reducing movement speed. Furthermore, left hand reaching was more accurate for far than near targets, whereas right hand reaching showed the opposite pattern. We discuss two possible hemispheric lateralization accounts of these findings.
\end{abstract}

Keywords Laterality · Handedness · Dominance · Reaching $\cdot$ Motor control

J. J. Adam (ه) · R. Müskens · S. Hoonhorst Faculty of Health, Medicine, and Life Sciences, Maastricht University, PO Box 616, 6200, MD, Maastricht, The Netherlands

e-mail: jos.adam@bw.unimaas.nl

J. Pratt

Department of Psychology, University of Toronto, Toronto M5S 3G3, Ontario, Canada

M. H. Fischer

School of Psychology, University of Dundee,

Dundee DD1 4HN, Scotland, UK

\section{Introduction}

Left and right hands often show robust performance differences that have been attributed to hemispheric specialization. Right-handers typically show right hand superiority in manual aiming, finger tapping, finger sequencing, and throwing, which has been ascribed to left hemispheric specialization for the organization and control of action. Left hand superiority in right-handers has been demonstrated for tasks that involve tactile discrimination and kinesthetic spatial judgments, which has been ascribed to right hemispheric superiority for spatial processing (for reviews, see Elliott and Chua 1996; Goble and Brown 2008).

A new, fascinating example of hand asymmetries has recently been provided by Gonzalez, Ganel, and Goodale (2006), who reported that grasping with the left hand is more sensitive to the effect of visual size illusions than grasping with the right hand. In their study, Gonzalez et al. asked left-handed and right-handed participants to pick up small objects presented in a visual context that made the objects look smaller or bigger than they actually were, using Ponzo and Ebbinghaus illusions. The key finding was that these illusory displays had a stronger effect on grip aperture in the left hand than in the right hand. Importantly, this was true for left-handers and right-handers. Emphasizing the fact that the right hand was able to escape the effect of the illusory visual context, Gonzalez et al. favored an interpretation in terms of a left hemisphere advantage for the visual control of action (e.g., Frey 2008). Alternatively, they also considered the well-known specialization of the right hemisphere for spatial processing (e.g., Kinsbourne 1978), which may explain the greater contextsensitivity of the left hand.

Both hemispheric specialization accounts predict that hand differences should extend beyond grasping to other 
visually guided actions such as reaching. de Grave et al. (2009), however, found no differences between left and right hand reaching in susceptibility to the Brentano illusion. Procedural and task differences between this and the Gonzalez et al. study make it difficult to draw strong conclusions about the generality of Gonzalez et al.'s findings. To shed more light on this issue, the present study investigated whether reaching movements executed with left and right hands are differentially sensitive to the influence of visual context. Instead of using visual illusions, however, we manipulated visual context by presenting targets either in isolation or in a linear array of placeholders.

The presence of placeholders, marking the possible target locations, has a dramatic influence on goal-directed reaching behavior. Relative to a situation without placeholders (i.e., the target appears in a blank field), the presence of a linear array of placeholders (one of which becomes the target) distinctively modulates the well-known relationship between movement time and distance-Fitts's Law (Fitts 1954): In the placeholder condition, movement time to the last target position is shorter than movement time to the second last target position, even though the last target position has a longer movement amplitude and a greater index of difficulty (Adam et al. 2006; Fischer et al. 2007; Pratt et al. 2007). This blatant violation of Fitts's Law shows that the presence of allocentric information (i.e., placeholders) is a powerful determinant of reaching behavior. Moreover, for the present issue, it also offers a new and useful paradigm to manipulate visual context.

In this study, we asked left-handed and right-handed participants to execute goal-directed reaching movements with left and right hands to targets appearing in placeholder and no-placeholder displays (see Fig. 1). Based on the findings of Gonzalez et al. (2006), we hypothesized that reaching with the left hand would show greater sensitivity to the presence of placeholders than reaching with the right hand, regardless of handedness. According to this hypothesis, a significant Hand (left, right) $\times$ Placeholders (present, absent) interaction should materialize for movement time and/or movement accuracy, independent of handedness.

\section{Methods}

\section{Participants}

Sixteen left-handed volunteers with a mean age of 22.9 years ( $\mathrm{SD}=5.6$, range $19-41$ years) and sixteen right-handed volunteers with a mean age of 21.9 years $(\mathrm{SD}=4.0$, range $18-35$ years) participated. Both
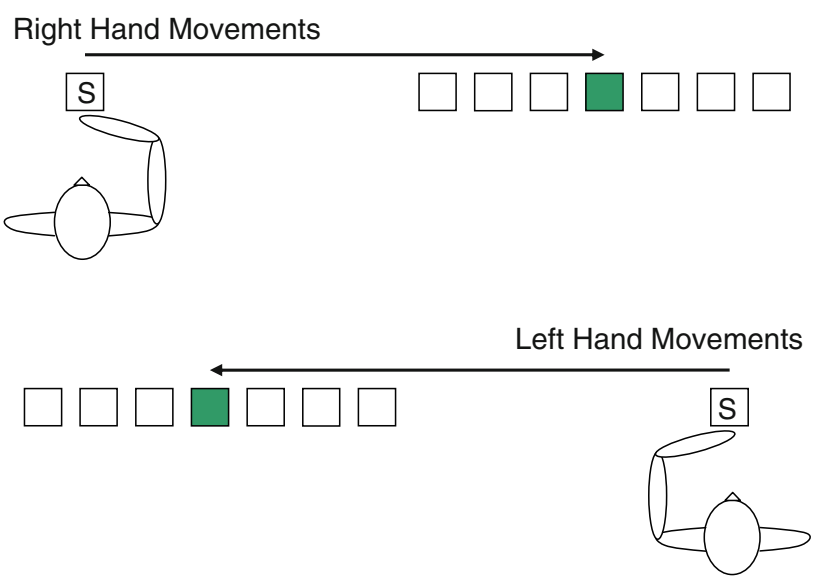

Fig. 1 Schematic overview of the start, target and body positions for movements with the right and left hand. Participants executed rapid hand movements from the start box to one of the seven target boxes (each $10 \times 10 \mathrm{~mm}$, separated by $5 \mathrm{~mm}$ ) with either the other boxes present (placeholder condition) or the other boxes absent (noplaceholder condition). The distance between the start box and first target box was $90 \mathrm{~mm}$ (side-to-side). The seven target locations were at increasing distances from the start location (i.e., 100, 115, 130, 145, 160,175 , and $190 \mathrm{~mm}$ )

handedness groups consisted of 4 males and 12 females. Handedness was assessed by the short version of the Edinburgh handedness questionnaire (Oldfield, 1971), which provides a laterality quotient (LQ): mean LQ was -77.4 and 84.7 , for left- and right-handers, respectively. All participants were students at Maastricht University, had normal or corrected to normal vision, received 10 euros for their participation, and gave written informed consent prior to their inclusion in the study. The data of one right-handed participant were not included in the data analyses because of exceptionally slow responses in all conditions.

Procedure and design

Participants made rapid aimed hand movements on a 19-inch touch screen to one of the seven target boxes that were horizontally arrayed at increasing distances from the start position (see Fig. 1). They stood in front of a table top on which the touch screen was placed horizontally (with a $10^{\circ}$ tilt upwards). All participants performed the aiming movements both with their left and right hands in two separate sessions on consecutive days. In each session, there were two display conditions (placeholder and no-placeholder). In the placeholder condition, the potential target positions were indicated by white placeholder boxes $(10 \times 10 \mathrm{~mm})$ that were continuously visible against a black background; the target signal was presented by coloring one of these placeholders green. In the no-placeholder condition, there were no placeholders, and the green target rectangle appeared by itself. With each hand, 
participants performed 112 test trials (16 for each target position), preceded by 28 practice trials, in each of the two placeholder conditions. Moreover, each series of 112 test trials was presented in four consecutive blocks of 28 trials, separated by a 25 -s pause. The order of the two placeholder conditions (within a session) and the order of the performing hand (across sessions) were counterbalanced, whereas the order of target positions within a series of 112 test trials was random.

When moving with the right hand, the target stimulus (and possible placeholders) appeared to the right of the start position. When moving with the left hand, the target stimulus (and possible placeholders) appeared to the left of the start position. Because participants were positioned such that the body midline was always in line with the start box, left and right hand movements were both abductive responses into ipsilateral space.

At the beginning of each trial, participants contacted the red start box with the index finger, which caused the start box to turn white and the green target stimulus to appear immediately and to stay on until the response was completed (touch down). If participants missed the target (touch down outside the target area $+1 \mathrm{~mm}$ extra margin), a visual error signal ("miss") was presented immediately for $1 \mathrm{~s}$. After $250 \mathrm{~ms}$ of the touch, or subsequent to the visual error signal, the target stimulus was removed. Then, after $500 \mathrm{~ms}$, the target box turned red again, signaling the start of the next trial. Participants were instructed to move their finger as quickly as possible to the target without making many errors.

\section{Analyses}

We calculated two measures of response time: (1) reaction time (RT), measured from the time the target stimulus appeared to the time when the start box was released; and (2) movement time (MT), measured from the time when the start box was released to when the target box was contacted. Furthermore, we calculated three different measures of response (end-point) accuracy: (1) percentage of target misses; (2) the mean of the absolute deviations from the target's center in the horizontal dimension (absolute error-AE); (3) the mean of the signed deviations from the target's center in the horizontal dimension (constant error-CE); negative values reflect undershoots, positive values reflect overshoots. RTs and MTs shorter than $100 \mathrm{~ms}$ or longer than $1,000 \mathrm{~ms}$ were regarded as outliers and excluded from data analysis. Using these criteria, $0.45 \%$ of the trials were removed. Mixed analyses of variance (ANOVA) were conducted on mean RT, MT, AE, CE and percentage of misses with handedness (left- and right-handers) as between-subjects variable, and hand (left, right), placeholders (present, absent), and target $(1,2,3,4,5,6,7)$ as within-subject variables. Whenever needed, the tests were adjusted for heterogeneity of variance and covariances using the Greenhouse-Geisser corrected significance values. An alpha level of .05 was used to determine statistical significance.

\section{Results}

\section{Reaction time}

Overall mean reaction time was $213 \mathrm{~ms}$. There were no statistically significant effects, even though RTs of the left hand tended to be somewhat longer than those of the right hand (217 ms vs. $208 \mathrm{~ms}$, respectively, $F(1,29)=4.04$, $P=.054){ }^{1}$

\section{Movement time}

The key finding was a significant Hand $\mathrm{x}$ Placeholder interaction $(F(1,29)=4.75, P<.05)$ shown in Fig. 2a,b. This interaction indicated that the presence of placeholders significantly slowed down left hand movements $(P<.01)$ but not right hand movements $(P>.7)$. This finding was similar for left- and right-handers, since the higher-order interaction with the factor handedness was not significant $(P>.2)$.

There was also a significant Target $\times$ Placeholder interaction $(F(6,174)=4.95, P<.001)$. This interaction reflects the well-established violation of Fitts's Law when placeholders are present (Adam et al. 2006; Fischer et al. 2007; Pratt et al. 2007): MTs to the last target (target 7) are shorter, not longer, than MTs to the second last target (target 6). Finally, there was a marginally significant interaction between Handedness and Hand $(F(1,29)=$ $3.47, P=.073)$, suggesting that right-handers tended to be faster with their right hand than with their left hand (472 ms vs. $495 \mathrm{~ms}$, respectively; $P=.053$ ), whereas lefthanders showed a very small, non-significant advantage for their dominant left hand (496 ms vs. $507 \mathrm{~ms}$, for left and right hands, respectively; $P=.48$ ).

\section{Absolute error}

There was a significant Hand $\mathrm{x}$ Placeholder interaction $(F(1,29)=5.85, P<.05)$ shown in Fig. $2 \mathrm{c}, \mathrm{d}$. This interaction indicated that the presence of placeholders

\footnotetext{
${ }^{1}$ Participants triggered onset of the target by contacting the start position. Given the known direction of the target, participants lifted their finger off the start location immediately to move in the required direction. Thus, RTs were generally very short and did not reliably capture motor planning processes.
} 
Fig. 2 Mean performance indices for left and right hand reaching movements as a function of target position and placeholder condition: (a, b) movement time $(\mathrm{ms})(\mathbf{c}, \mathbf{d})$ absolute error $(\mathrm{mm})(\mathbf{e}, \mathbf{f})$ constant error (mm). Note, for constant error, negative values reflect undershoots, positive values reflect overshoots relative to the target's midpoint in the principal direction of motion
Left Hand
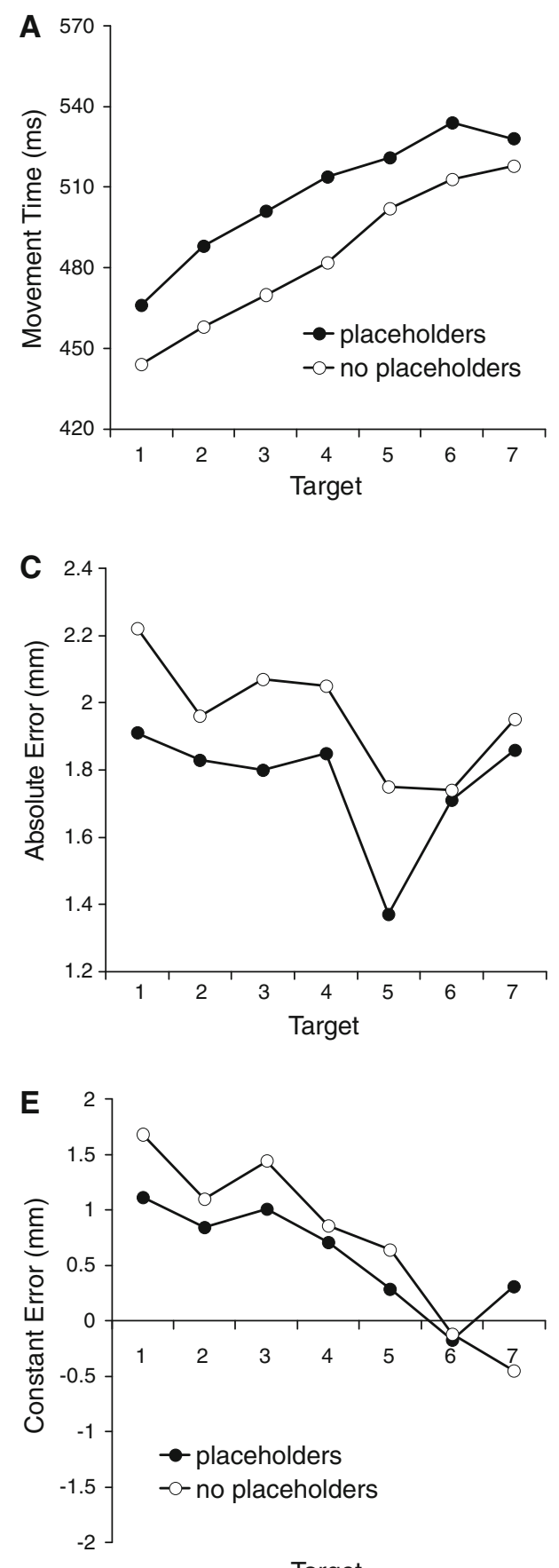

Right Hand
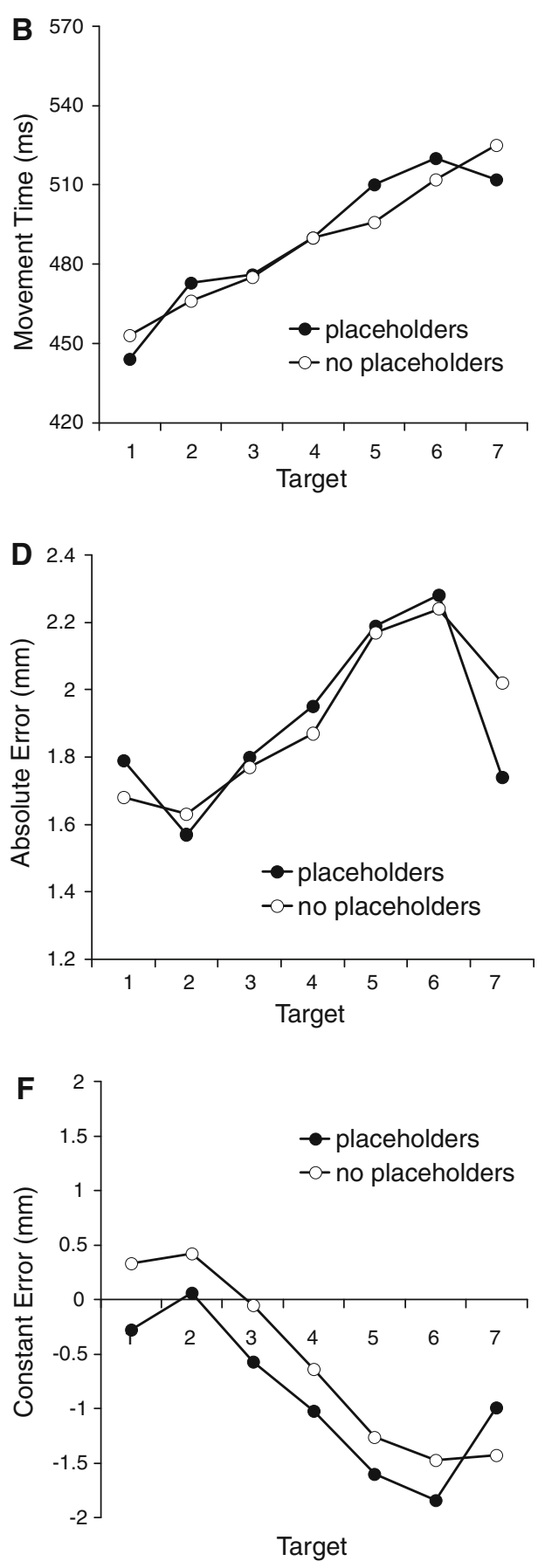

substantially reduced AE for left hand reaching $(P<.001)$ but not for right hand reaching $(P>.8)$. This effect tended to be stronger for left-handers than for right-handers, but this finding was statistically not reliable $(F(1,29)=3.60$, $P=.068$ ).

There was also a significant Hand $\times$ Target interaction $(F(6,174)=22.74, P<.001)$. This interaction reflected opposite effects of target position (distance) on left and right hand movements: whereas left hand movements were more accurate than right hand movements for far targets (i.e., targets 5 and $6 ; P<.001$ ), right hand movements were more accurate than left hand movements for near targets (i.e., targets 1 and $2, P<.001$ ). The significant three-way interaction with the factor Placeholder $(F(7,174)=3.04, P<.01)$ indicated that the presence of placeholders eliminated the advantage of the left hand at near positions and increased the advantage of the right hand at far positions. 


\section{Constant error}

The main effect of Target $(F(6,174)=121.65, P<.001)$ reflected a range effect (i.e., overshoots for near targets and undershoots for far-away targets). The main effect of Hand $(F(1,29)=125.66, P<.001)$ reflected a bias for the left hand to produce overshoots and a bias for the right hand to produce undershoots (see Fig. 2e,f). The significant Target $\mathrm{x}$ Hand interaction $(F(6,174)=11.31, P<.001)$ indicated that the difference in CE between the two hands was smallest for near targets (i.e., targets 1 and 2).

Target Misses. Overall, left and right hand movements missed the target about equally often (4.8 vs. $4.2 \%$, respectively, $P>.3$ ). However, the significant Target $\mathrm{x}$ Hand interaction $(F(6,174)=7.15, P<.001)$ indicated that the left hand missed the target significantly more often than the right hand at near positions (targets 1, 2, and 3), whereas the opposite tended to be true for far targets. Finally, the significant Hand $\mathrm{x}$ Handedness interaction $(F(1,29)=4.60, P<.05)$ indicated that right-handers missed the target less often with the right hand than with the left hand ( 3.1 vs. $4.8 \%$ misses, respectively; $P<.01$ ), whereas left-handers showed a small, non-significant advantage for the left hand over the right hand (4.7 vs. $5.4 \%$, respectively; $P=.51$ ).

\section{Discussion}

The key finding of this study was that reaching movements with the left hand, but not those with the right hand, were significantly modulated by the presence of placeholders, which improved terminal accuracy at the cost of movement speed. This was true for both left- and right-handed participants. These outcomes are consistent with the findings of Gonzalez et al. (2006) despite the fact that we examined reaching not grasping, and used a veridical instead of an illusory visual context. Thus, our findings provide strong converging evidence for Gonzalez et al.'s hypothesis that left and right hand movements are differentially sensitive to the effects of visual context.

How to explain the hand laterality effect? The fact that it occurred regardless of handedness strongly suggests an interpretation in terms of hemispheric specialization. Gonzalez et al. (2006) favored an interpretation in terms of the segregation of action and perception systems in left and right hemispheres, respectively. This account can also explain why the right hand was more accurate for near than far targets, whereas the left hand was more accurate for far than near targets. In this view, action is tuned to space near the hand, because space near the hand is of primary importance for action control. Everyday perception, in contrast, is often directed to objects in the periphery, which may explain the superiority of the more "ventrally guided" left hand for targets farther away.

An alternative interpretation of the present results, however, is also possible. In an excellent review of hemispheric specialization in the motor domain, Serrien, Ivry, and Swinnen (2006) discerned several key functions of the right hemisphere, including its well-established preference to process global features of the display, which contrasts with the left hemisphere's preference to process local features (e.g., Ivry and Robertson 1998). This hemispheric difference in global versus local processing might explain the left hand's sensitivity and right hand's immunity to visual context information.

Importantly, Serrien et al. (2006) also highlighted two additional, but often neglected, specializations of the right hemisphere. First, they noticed that the right hemisphere is crucially involved in the closed-loop control of movements (which involves online processing of feedback information), whereas the left hemisphere is preferentially engaged in open-loop control (which is based on pre-specified motor programs; e.g., Haaland and Harrington 1989). Second, they noted that the right hemisphere is specialized in controlling limb position, whereas the left hemisphere is specialized in controlling limb dynamics (e.g., Sainburg 2002; Winstein and Pohl 1995). These two specializations are convergent in that open-loop left hemisphere specialization might be limited to feed-forward specification of movement dynamics, whereas closed-loop right hemisphere specialization includes sensory-based mechanisms that control final limb position (Bagesteiro and Sainburg 2003).

This latter conceptualization provides an alternative framework to interpret our Absolute Error data, which showed a left hand superiority for far targets and a right hand superiority for near targets. The behavior of the right hand can be explained by an open-loop control mode, as movements covering greater distances are driven by stronger neuromotor impulses, which result in concomitant increases in variability (Meyer et al. 1988). The behavior of the left hand is instead consistent with closed-loop control, which optimizes end-point accuracy (Meyer et al. 1988). This is especially effective for "long distance" movements, because these movements generally are of longer duration, thus providing more time to implement time-consuming feedback processes.

When reaching with the left hand, the presence of placeholders seems to boost online control even for nearby targets, thus eliminating the advantage that the right hand enjoys for these targets (see Fig. 2c,d). Hence, one might conjecture that the right hemisphere's global processing bias and its superior online control are related phenomena in that the former may promote the latter. In this view, a broad, global processing perspective allows 
non-target information to be processed, thereby creating visuomotor competition that needs to be resolved (e.g., Tipper et al. 1992). The present movement time and endpoint data are consistent with the notion of greater executive control during movement execution of the left hand, especially when targets appear in a cluttered environment.

The notion that the right hemisphere is specialized in feedback-based online control of movements might also explain why de Grave et al. (2009) failed to find hand differences in susceptibility to an illusory visual context. The critical factor here might be the visibility of the target during movement execution. Whereas in the study of Gonzalez et al. (2006), the target remained visible throughout the movement, in the de Grave et al. study, the target was removed at movement onset, thus eliminating the possibility of online movement corrections based on visual feedback.

Finally, both hands in the present study showed a range effect, but overall, the left hand was biased to produce overshoots, whereas the right hand was biased to produce undershoots. Since both hands moved in opposite directions, these two findings can be understood as indicating an overall leftward bias in reaching, which may reflect pseudoneglect. This phenomenon exists in healthy individuals and causes the left side extent of a stimulus to be overestimated relative to the right, as has been demonstrated in line bisection tasks (e.g., Jewell and McCourt 2000).

In conclusion, the current study revealed two important new findings that support a strong dissociation between left and right hand reaching regardless of handedness. First, left hand, but not right hand, reaching was influenced by the presence of placeholders, which reduced movement speed but improved movement accuracy. Second, left hand reaching was more accurate for far than for near targets, whereas right hand reaching was more accurate for near than for far targets. These two hand laterality effects are consistent with a right hemispheric specialization for, respectively, global spatial information processing and closed-loop control of final hand position.

Open Access This article is distributed under the terms of the Creative Commons Attribution Noncommercial License which permits any noncommercial use, distribution, and reproduction in any medium, provided the original author(s) and source are credited.

\section{References}

Adam JJ, Mol R, Pratt J, Fischer MH (2006) Moving farther but faster: an exception to Fitts's law. Psychol Sci 17:795-799

Bagesteiro LB, Sainburg RL (2003) Nondominant arm advantages in load compensation during rapid elbow joint movements. J Neurophysiol 90:1503-1513

de Grave DDJ, Brenner E, Smeeets JBJ (2009) The Brentano illusion influences goal-directed movements of the left and right hand to the same extent. Exp Brain Res 193:421-427

Elliott D, Chua R (1996) Manual asymmetries in goal-directed movement. In: Elliot D, Roy EA (eds) Manual asymmetries in motor performance. CRC Press, Boca Raton, pp 143-158

Fischer MH, Pratt J, Adam JJ (2007) On the timing of reference frames for action control. Exp Brain Res 183:127-132

Fitts PM (1954) The information capacity of the human motor system in controlling the amplitude of movement. J Exp Psychol 47:381-391

Frey SH (2008) Tool use, communicative gesture and cerebral asymmetries in the modern human brain. Philos Trans R Soc Lond B 363:1951-1957

Goble DJ, Brown SH (2008) The biological and behavioral basis of upper limb asymmetries in sensorimotor performance. Neurosci Biobehav Rev 32:598-610

Gonzalez CL, Ganel T, Goodale MA (2006) Hemispheric specialization for the visual control of action is independent of handedness. J Neurophysiol 95:3496-3501

Haaland KY, Harrington DL (1989) Hemispheric control of the initial and corrective components of aiming movements. Neuropyschologia 27:961-969

Ivry RB, Robertson LC (1998) The two sides of perception. MIT Press, Cambridge

Jewell G, McCourt ME (2000) Pseudoneglect: a review and metaanalysis of performance factors in line bisection tasks. Neuropsychologia 38:93-110

Kinsbourne M (1978) Asymmetrical function of the brain. Cambridge University Press, Cambridge

Meyer DE, Abrams RA, Kornblum S, Wright CE, Smith JEK (1988) Optimality in human motor performance: ideal control of rapid aimed movements. Psychol Rev 95:340-370

Oldfield RC (1971) The assessment and analysis of handedness: the Edinburgh inventory. Neuropsychologia 9:97-113

Pratt J, Adam JJ, Fischer MH (2007) Visual layout modulates Fitts's law: the importance of first and last positions. Psychon Bulletin Rev 14:350-355

Sainburg RL (2002) Evidence for a dynamic-dominance hypothesis of handedness. Exp Brain Res 142:241-258

Serrien DJ, Ivry RB, Swinnen SP (2006) Dynamics of hemispheric specialization and integration in the context of motor control. Nature Rev Neurosci 7:160-167

Tipper SP, Lortie C, Baylis GC (1992) Selective reaching: evidence for action-centered attention. J Exp Psychol Hum Percept Perform 18:891-905

Winstein CJ, Pohl PS (1995) Effects of unilateral brain damage on the control of goal-directed hand movements. Exp Brain Res 105:163-174 